\title{
EFETIVIDADE DE UM PROGRAMA DE APRIMORAMENTO VOCAL PARA PROFESSORES
}

\section{Effectiveness of a vocal improvement program for teachers}

\author{
Karen Fontes Luchesi ${ }^{(1)}$, Lucia Figueiredo Mourão ${ }^{(2)}$, Satoshi Kitamura ${ }^{(3)}$
}

\section{RESUMO}

Objetivo: analisar parâmetros fonoarticulatórios de professores, pré e pós-programa de aprimoramento vocal. Método: um programa de aprimoramento vocal foi oferecido numa escola estadual do município de Campinas, São Paulo, Brasil. Treze professores aceitaram participar do programa. Devido ao critério de exclusão, apenas cinco foram selecionados para as análises. Os sujeitos foram previamente submetidos à avaliação laringológica. Foram realizados 12 encontros semanais de 1 hora e 30 minutos na própria escola. No primeiro e no último encontro os sujeitos gravaram três "frases-veículo". As gravações foram submetidas à análise perceptivo-auditiva (para avaliação do pitch, da modulação e da articulação) e acústica (para avaliação da freqüência fundamental, da extensão de freqüência e dos dois primeiros formantes). Os dados foram submetidos à análise estatística. Resultados: mesmo com o pequeno número de sujeitos, os resultados do presente estudo, revelaram ampliação significante da extensão de freqüência, indicando maior uso deste recurso expressivo pós-aprimoramento, além do aumento estatisticamente significante de $F_{1}$ nas vogais /i/ e /u/ pós-intervenção, sugerindo melhora no ajuste articulatório. Conclusões: não foram observadas modificações no pitch, modulação e articulação avaliados por meio da análise perceptivo-auditiva, bem como da freqüência fundamental pós-intervenção.

DESCRITORES: Voz; Docentes; Saúde do Trabalhador; Treinamento da Voz; Prevenção de Doenças

\section{INTRODUÇÃO}

O freqüente aparecimento de alterações vocais em professores tem levado pesquisadores a destacar a grande importância das intervenções de

(1) Fonoaudióloga; Mestre e Doutoranda em Saúde Coletiva pela Faculdade de Ciências Médicas da Universidade Estadual de Campinas.

(2) Fonoaudióloga; Docente do Curso de Fonoaudiologia da Faculdade de Ciências Médicas, Universidade Estadual de Campinas; Doutora em Ciências pela Universidade Federal de São Paulo.

(3) Médico do Trabalho; Docente do Departamento de Medicina Preventiva e Social (Área de Saúde do Trabalhador), Faculdade de Ciências Médicas, Universidade Estadual de Campinas; Doutor em Ciências Médicas pela Faculdade de Ciências Médicas da Unicamp.

Trabalho realizado na Universidade Estadual de Campinas. Artigo baseado na dissertação de mestrado de Karen Fontes Luchesi, "Desenvolvimento de um programa de aprimoramento vocal numa escola de ensino fundamental do município de Campinas: estudo de caso", apresentada à Faculdade de Ciências Médicas da Universidade Estadual de Campinas em 2008.

Conflito de interesses: inexistente caráter preventivo ${ }^{1-19}$. Dentre estas ações, estão as intervenções educativas voltadas para os cuidados com a voz e à adequação fonoarticulatória, visando uma produção vocal mais saudável. Apesar do alto índice de alterações vocais em professores, poucos estudos avaliaram e discutiram intervenções desenvolvidas para prevenir e tratar a disfonia nesses profissionais. Os estudos realizados com este intuito observaram melhora na qualidade e nos sintomas vocais pós-intervenção $0^{2-4,10,14}$.

Pesquisas realizadas nos últimos dez anos observam que a falta de conhecimento sobre a fonação, o despreparo vocal e as condições de trabalho são fatores bastante relevantes para a instalação de alterações vocais e o afastamento de muitos professores de seus trabalhos ${ }^{5,6,13,16}$.

O objetivo do presente artigo foi analisar parâmetros, vocais e articulatórios de professores, pré e pós-programa de aprimoramento vocal. 


\section{MÉTODO}

Um programa de aprimoramento vocal foi oferecido numa escola estadual de ensino fundamental ciclo I (antigas 1a. a 4a. série), com 26 professores, situada no Distrito de Saúde Leste do município de Campinas, estado de São Paulo, Brasil. Utilizou-se como critério de inclusão ser professor da escola e ter concordado com a participação. Foi estabelecido como critério de exclusão a participação em menos de 65\% (8/12) dos encontros. Treze professores aceitaram participar do programa, no entanto, devido ao critério de exclusão apenas cinco foram selecionados para as análises.

Com o objetivo de conhecer as condições laríngeas dos sujeitos, esses foram previamente submetidos a uma avaliação laringológica no Hospital das Clínicas da Unicamp, na Área da Otorrinolaringologia. Os cinco professores selecionados para participar do estudo, consentiram em submeter-se à avaliação laringológica (Tabela 1).

Tanto os professores com alteração quanto os professores sem alteração laríngea puderam participar do programa de aprimoramento vocal.

Baseado na disponibilidade dos professores foram organizados dois grupos de 6 e 7 sujeitos. Foram realizados 12 encontros semanais de 1 hora e 30 minutos, na biblioteca e na sala de vídeo da escola, todos coordenados pela mesma fonoaudióloga. Os encontros abordaram itens reconhecidos como fundamentais para uma fonoarticulação adequada e saudável ${ }^{1,3,20}$, a saber: noções de anatomia e fisiologia fonatória, saúde vocal (hábitos e cuidados), respiração, coordenação pneumofonoarticulatória, tensão fonatória, articulação, velocidade e modulação da fala, ressonância, projeção vocal, expressividade verbal e não-verbal, aquecimento e desaquecimento vocal. Cada encontro abordou de um a dois desses itens, que foram desenvolvidos em aulas expositivas com utilização de recursos áudios-visuais, apresentação oral, diálogo entre os participantes, dinâmicas de grupo e aplicação de técnicas vocais (Figura 1).

Preconizou-se a realização das técnicas vocais nos encontros e no cotidiano dos sujeitos. Foi entregue aos sujeitos material impresso com breve explanação sobre os assuntos abordados e descrição das técnicas vocais utilizadas.

No primeiro encontro dos grupos os participantes responderam a um formulário com os dados: idade, tempo de profissão, formação acadêmica, carga horária semanal e queixa vocal (caso houvesse). No primeiro e no último encontro os sujeitos gravaram três frases-veículo: "eu quero um /pa'papa/ legal pra mim", "eu quero um /pa'pipa/ legal pra mim" e "eu quero um /pa'pupa/ legal pra mim". Estas foram utilizadas como veículo para palavras-alvo criadas de modo que as vogais, vértices do triângulo vocálico (/a/, /i/, /u/), estivessem sempre em posição tônica e precedidas pela oclusiva /p/. Tanto na pré quanto na pós-intervenção os sujeitos foram orientados a ler as frases de maneira espontânea.

Apesar do número reduzido de sujeitos, as análises foram baseadas em 150 amostras de fala, pois consistiram em cinco repetições de três frases por cinco sujeitos em dois momentos, pré e pós-intervenção.

A fala dos sujeitos foi registrada pelo software livre Praat (www.praat.org) a uma taxa de amostragem de $44100 \mathrm{~Hz}$. Utilizando notebook Toshiba com placa de som externa "Móbile Pré" da marca M-áudio, em sala silenciosa da própria escola, com microfone unidirecional da marca Shure a, aproximadamente, $10 \mathrm{~cm}$ de distância da boca.

O mesmo material de fala foi submetido à análise perceptivo-auditiva e acústica. Para a análise perceptivo-auditiva, três fonoaudiólogos especialistas em voz pela mesma instituição, com prática clínica em voz de 12 a 15 anos, avaliaram individualmente os parâmetros: pitch, modulação e articulação. Os especialistas receberam as amostras distribuídas em arquivos randomizados, sem a identificação dos sujeitos ou do momento em que foram gravados. Foram orientados a marcar um traço vertical cruzando uma escala analógica visual, ou seja, uma linha horizontal de dez centímetros com limites à direita e à esquerda, onde melhor representasse a sua percepção para cada item avaliado (Figura 2). A extremidade esquerda foi caracterizada como "inadequada" (valores próximos de zero) e a direita, como "adequada" (valores próximos de 10). Posteriormente, foi encontrado um correspondente numérico (em centímetros) para cada parâmetro avaliado utilizando-se de uma régua milimétrica. Para obter um valor comum aos três avaliadores, foram calculadas as médias e os desvios-padrão dos correspondentes numéricos.

A análise acústica foi realizada por meio do software Praat e teve por objetivo analisar os parâmetros: freqüência fundamental $\left(f_{0}\right)$, extensão de freqüência e os dois primeiros formantes. Para medir a freqüência fundamental e os formantes, selecionaram-se as vogais tônicas das palavrasalvo. Sendo que, para delinear o início e o fim das vogais, tomou-se por base o aparecimento e o desaparecimento do padrão formântico, principalmente do primeiro $\left(F_{1}\right)$ e do segundo formante $\left(F_{2}\right)$. Obteve-se a média da freqüência fundamental e dos dois primeiros formantes de cada vogal. Para calcular a extensão de freqüência foram 
EPATAS:

1) Divulgação direta e indireta do programa. Divulgação direta: exposição oral aos professores em Hora de Trabalho Pedagógico Coletivo (HTPC). Divulgação Indireta: distribuição e afixação de folders sobre o programa.

2) Encaminhamento e realização de exames laringoscópicos.

3) Formação dos grupos e realização dos encontros:

$1^{\circ}$ ENCONTRO: Apresentação do programa, preenchimento do formulário e gravação pré-aprimoramento.

$2^{\circ}$ ENCONTRO: Noções de anatomia e fisiologia do sistema fonatório (utilização de recursos áudio-visuais imagens e vídeos), principais causas e sintomas de disfonias.

$3^{\circ}$ ENCONTRO: Discussão sobre saúde vocal do professor.

i. Cuidados na vida diária: hábitos, alimentação etc; cuidados quanto ao uso da voz: quanto ao ambiente, à postura, à dinâmica em sala de aula etc.

$4^{\circ}$ ENCONTRO: Reflexão e discussão sobre psicodinâmica vocal, expressão corporal e facial.

ii. Tipos de voz e as impressões que causam no aluno.

iii. Dinâmica de grupo: dramatizações relacionando tipos vocais e situações de aula. Ex.: Representação de diálogo entre aluno e professor, experimentando conversas com diferentes intensidades, pitch, velocidades de fala e tipos articulatórios. Reflexão sobre as impressões que podem causar nos alunos.

iv. Discussão sobre o uso de recursos expressivos não-verbais como gestos, movimentação corporal e expressão facial.

$5^{\circ}$ ENCONTRO: Respiração e Intensidade vocal.

v. Explicação e percepção dos tipos respiratórios e coordenação pneumo-fono-articulatória.

vi. Aplicação de técnica vocal: técnica de sons fricativos.

vii. Dinâmica de grupo: representação de diálogo entre aluno e professor, experimentando diferentes intensidades vocais (adequada, aumentada e diminuída), discussão sobre a repercussão da utilização de cada uma delas nos alunos.

$6^{\circ}$ ENCONTRO - Alívio da tensão fonatória e relaxamento.

viii. Percepção dos professores sobre seus pontos de tensão.

ix. Alongamento cervical: rotação de cabeça e ombros e movimentos de contra-resistência. Relaxamento facial: movimentos circulares com os dedos indicadores e médios em toda a face.

x. Aplicação de técnicas vocais: técnica de manipulação digital da laringe, técnica de bocejo-suspiro, técnica de firmeza glótica e técnica de vibração.

$7^{\circ}$ e $8^{\circ}$ ENCONTRO: Articulação e velocidade de fala.

xi. Dinâmica: "Telefone sem fio" (sentados em círculo, um participante tinha que fazer o próximo compreender uma palavra, só com a articulação dos sons, sem nenhuma emissão). Discussão sobre a importância de uma articulação precisa no ensino.

xii. Percepção da diferença entre os pontos e os modos de articulação dos fonemas da Língua Portuguesa, por meio da produção de vogais e consoantes isoladas e da leitura de trava-línguas.

xiii. Aplicação de técnicas vocais: técnica de sons fricativos associados a vogais, técnica de rotação de língua no vestíbulo associada a som nasal, técnica de estalo de língua, técnica mastigatória, técnica de sobrearticulação e técnica de voz salmodiada.

$9^{\circ}$ ENCONTRO: Modulação.

xiv. Discussão sobre a importância da modulação no ensino.

xv. Aplicação de técnicas vocais: técnica de voz salmodiada e técnica de vibração associada à variação de freqüência.

xvi. Leitura de diálogos, extraídos de livros clássicos da Literatura Brasileira.

xvii. Dinâmicas de grupo: Participantes variando suas vozes para causar diferentes impressões no ouvinte (medo, nervosismo, sedução, infantilidade, agitação, monotonia etc).

$10^{\circ}$ ENCONTRO: Ressonância e projeção vocal.

xviii. Discussão sobre a importância de uma voz projetada para o professor, e a relação existente entre apoio respiratório, condições laríngeas, articulação, ressonância e projeção vocal.

xix. Aplicação de técnicas vocais: técnica de sons nasais, técnica mastigatória, técnica de vibração associada à vogais, técnica de estalo nasal associado a som nasal.

$11^{\circ}$ ENCONTRO: Aquecimento e Desaquecimento vocal.

xx. Reflexão sobre a importância do aquecimento e do desaquecimento vocal. Orientação para a realização do aquecimento vocal cerca de 15 minutos antes da aula (elegendo de dois a três exercícios aprendidos durante os encontros). Foi orientada a realização do desaquecimento vocal logo após a aula, realizando-o por cerca de cinco minutos, com as técnicas de bocejo-suspiro e glissando descendente.

$12^{\circ}$ ENCONTRO: Discussão sobre o programa e os temas abordados. Gravações pós-aprimoramento.

\section{Figura 1 - Etapas do Programa de Aprimoramento Vocal desenvolvido}

medidos os pontos máximos e mínimos de freqüência das frases inteiras e calculada a diferença entre estes valores.

Os parâmetros fonoarticulatórios avaliados neste estudo foram selecionados com o intuito de estabelecer um paralelo entre a análise perceptivoauditiva e a análise acústica, no qual o pitch corresponde à sensação psicofísica da freqüência fundamental, a modulação à extensão de freqüência e a articulação aos dois primeiros formantes (que 
AMOSTRA №:

Assinale um traço vertical nas linhas abaixo (escala analógica visual de $10 \mathrm{~cm}$ ), que caracterize sua opinião para as amostras de fala apresentadas. O lado direito representa "alteração inexistente" ou "adequada" e o lado esquerdo representa "completamente alterada" ou "inadequada".

1) PITCH (padrão optimal: adequado para idade, sexo e para o contexto).

2) MODULAÇÃo (padrão optimal: freqüência variável, sem monotonia e/ou repetições).

3) ARTICULAÇÃo DE FALA (padrão optimal: sem distorções, flexível, sem tensões aparentes).

\section{Figura 2 - Protocolo para avaliação perceptivo-auditiva.}

correspondem, respectivamente, à movimentação vertical e horizontal da língua ${ }^{21}$.

Procurou-se selecionar aspectos que pudessem ser modificados mesmo em professores sem alteração vocal. Baseado no pressuposto de que na população sem alterações laríngeas o mais importante é melhorar aspectos da produção fonoarticulatória, destacando-se a importância da articulação, modulação e projeção vocal para a produção de uma voz saudável e expressiva. Houve a preocupação em selecionar para a avaliação parâmetros que haviam sido tema dos encontros da intervenção, que pudessem ser analisados perceptiva e acusticamente, intencionando a complementação. Não puderam ser selecionados parâmetros como loudness ou parâmetros de perturbação da onda sonora devido à falta de tratamento acústico no local de coleta.

Esse estudo foi aprovado pelo Comitê de Ética da Faculdade de Ciências Médicas da Universidade Estadual de Campinas (Unicamp) (CEP №484/2007). Todos os sujeitos que concordaram em participar deram sua anuência assinando o Termo de Consentimento Livre e Esclarecido.

Para caracterizar a distribuição dos dados foi empregada uma análise estatística descritiva. Devido ao número de amostras de fala analisadas (150), os dados foram apresentados por meio das médias e dos desvios-padrão dos valores obtidos para cada aspecto avaliado. Verificou-se a normalidade na distribuição dos dados pela aplicação do teste de Kolmogorov-Smirnov. Para a comparação dos valores obtidos pré e pós-intervenção de cada sujeito foi aplicado o teste $t$ de Student para amostras pareadas. A fim de quantificar o grau de relação entre os resultados da avaliação perceptivo-auditiva e da avaliação acústica foi calculado o coeficiente de correlação de Pearson. Foi considerada a probabilidade $(p)$ menor que 0,05 para indicar significância estatística.

\section{RESULTADOS}

Treze professores aceitaram participar do programa, no entanto, devido ao critério de exclusão apenas cinco foram selecionados para as análises (Tabela 1).

É importante levar em consideração que os resultados aqui apresentados são válidos apenas para a população estudada e que não podem ser generalizados devido ao número insuficiente de sujeitos considerados para análise. A presença de altos valores de desvio padrão para os parâmetros analisados (Tabelas 2 e 3) pode ser explicada por essa razão.

Os resultados das análises perceptivo-auditiva e acústica pré e pós-intervenção foram apresentados nas Tabelas 2 e 3, respectivamente.

$\mathrm{Na}$ avaliação perceptivo-auditiva observou-se o aumento das médias pós-intervenção dos três parâmetros avaliados, e a diminuição do desvio-padrão dos parâmetros modulação e articulação pós-intervenção. No entanto, não houve significância estatística para as diferenças pré e pós-intervenção em nenhum dos parâmetros (Tabela 2).

Em relação à análise acústica, houve um aumento da média de $f_{0}$ para as vogais /i/ e / $u /$ e uma redução sistemática dos desvios-padrão pósintervenção das três vogais. No entanto, o tamanho da amostra não permitiu nenhuma conclusão de aumento ou diminuição de $f_{0}$ para nenhuma vogal. Aumento estatisticamente significante foi observado na extensão de freqüência e no primeiro formante das vogais /i/ e /u/ pós-intervenção (Tabela 3). 
Tabela 1 - Descrição dos casos quanto ao gênero, idade, tempo de profissão, formação acadêmica, carga horária semanal, queixa vocal, avaliação laringológica e participação no programa de aprimoramento vocal

\begin{tabular}{|c|c|c|c|c|c|c|c|c|c|}
\hline Sujeito & Gênero & $\begin{array}{l}\text { Idade } \\
\text { (anos) }\end{array}$ & $\begin{array}{c}\text { Tempo } \\
\text { de } \\
\text { Profissão } \\
\text { (anos) } \\
\end{array}$ & $\begin{array}{l}\text { Formação } \\
\text { Acadêmica }\end{array}$ & $\begin{array}{c}\text { Carga } \\
\text { Horária } \\
\text { (hora-aula) }\end{array}$ & $\begin{array}{c}\text { Queixa } \\
\text { Vocal }\end{array}$ & Av. Laring. & $\begin{array}{c}\text { Hipótese } \\
\text { Diagnóstic } \\
\text { a }\end{array}$ & $\begin{array}{l}\text { № } \\
\text { Enc. } \\
\text { Part. }\end{array}$ \\
\hline 1 & $\mathrm{~F}$ & 26 & 2 & Letras & 20 & Sim & Sim & Nódulos & 12 \\
\hline 2 & $\mathrm{~F}$ & 50 & 9 & Ed. Física & 20 & Sim & Sim & Monocordite & 12 \\
\hline 3 & $F$ & 49 & 6 & Ed. Física & 12 & Não & Sim & $\begin{array}{l}\text { Edema de } \\
\text { Reinke }\end{array}$ & 10 \\
\hline 4 & $F$ & 34 & 13 & Pedagogia & 40 & Não & Sim & $\begin{array}{c}\text { Sem } \\
\text { alteração }\end{array}$ & 9 \\
\hline 5 & $\mathrm{~F}$ & 50 & 24 & Pedagogia & 18 & Não & Sim & $\begin{array}{c}\text { Sem } \\
\text { alteração }\end{array}$ & 8 \\
\hline
\end{tabular}

Abreviaturas: F = Feminino; $M$ = Masculino; Ed. Física = Educação Física; Av. Laring. = Avaliação Laringológica; № Enc. Part. = Número de Encontros que Participou.

Tabela 2 - Valores médios, desvios-padrão e comparações pré e pós-intervenção dos parâmetros pitch, modulação e articulação, em cm, obtidos com a utilização da escala analógica visual de 100mm

\begin{tabular}{lccccccc}
\hline \multirow{2}{*}{ PARÂMETROS } & \multirow{2}{*}{$\mathbf{N}$} & \multicolumn{2}{c}{ PRÉ } & \multicolumn{2}{c}{ PÓS } & DIF. & T-STUDENT \\
\cline { 3 - 5 } \cline { 5 - 6 } & & MÉDIA & D.P. & MÉDIA & D.P. & P/P & $\mathbf{p}$ \\
\hline Pitch & 5 & 9,207 & 0,65 & 9,267 & 0,69 & 0,06 & 0,877 \\
Modulação & 5 & 8,14 & 1,41 & 8,96 & 0,73 & 0,82 & 0,357 \\
Articulação & 5 & 8,793 & 1,04 & 9,253 & 0,65 & 0,46 & 0,451 \\
\hline
\end{tabular}

Legenda: D.P. = Desvio-Padrão; DIF. P/P = Diferença entre as médias Pós/Pré.

Tabela 3 - Valores médios, desvios-padrão e comparações pré e pós-intervenção das medidas de freqüência fundamental, extensão de freqüência e primeiro e segundo formantes, em $\mathrm{Hz}$

\begin{tabular}{|c|c|c|c|c|c|c|c|c|}
\hline \multirow{2}{*}{ PARÂMETROS } & \multirow{2}{*}{$\mathbf{N}$} & \multirow{2}{*}{ VOGAIS } & \multicolumn{2}{|c|}{ PRÉ } & \multicolumn{2}{|c|}{ PÓS } & \multirow{2}{*}{$\begin{array}{l}\text { DIF. } \\
\text { P/P }\end{array}$} & \multirow{2}{*}{$\begin{array}{c}\text { T-STUDENT } \\
p\end{array}$} \\
\hline & & & MÉDIA & D.P. & MÉDIA & D.P. & & \\
\hline \multirow{3}{*}{$\begin{array}{l}\text { Freqüência } \\
\text { Fundamental }(\mathrm{Hz})\end{array}$} & 5 & $/ a /$ & 175,955 & 21,426 & 166,628 & 18,681 & $-9,327$ & 0,241 \\
\hline & 5 & /i/ & 200,858 & 19,514 & 209,024 & 6,957 & 8,166 & 0,333 \\
\hline & 5 & $/ \mathrm{u} /$ & 197,922 & 20,913 & 217,357 & 19,375 & 19,44 & 0,11 \\
\hline \multirow{3}{*}{$\begin{array}{l}\text { Extensão de } \\
\text { Freqüência (Hz) }\end{array}$} & 5 & /a/ & 115,831 & 22,551 & 144,103 & 58,874 & 28,27 & 0,278 \\
\hline & 5 & /i/ & 114,366 & 23,455 & 167,045 & 30,776 & 52,68 & $0,03^{*}$ \\
\hline & 5 & $/ \mathrm{u} /$ & 113,468 & 27,18 & 163,171 & 28,183 & 49,7 & $0,001^{*}$ \\
\hline \multirow{3}{*}{$\begin{array}{l}\text { Primeiro Formante } \\
(\mathrm{Hz})\end{array}$} & 5 & $/ \mathrm{a} /$ & 821,958 & 152,205 & 806,27 & 109,037 & $-15,688$ & 0,712 \\
\hline & 5 & /i/ & 276,958 & 25,596 & 305,64 & 21,377 & 28,68 & $0,044^{*}$ \\
\hline & 5 & $/ \mathrm{u} /$ & 348,69 & 35,316 & 407,528 & 30,762 & 58,84 & $0,009^{*}$ \\
\hline \multirow{3}{*}{$\begin{array}{l}\text { Segundo Formante } \\
(\mathrm{Hz})\end{array}$} & 5 & $/ \mathrm{a} /$ & 1416,532 & 53,718 & 1381,329 & 34,402 & $-35,203$ & 0,133 \\
\hline & 5 & /i/ & 2511,092 & 107,36 & 2496,644 & 163,937 & $-14,448$ & 0,706 \\
\hline & 5 & $/ \mathrm{u} /$ & 840,127 & 104,664 & 866,618 & 158,937 & 26,49 & 0,748 \\
\hline
\end{tabular}

Legenda: D.P. = Desvio-Padrão; DIF. P/P = Diferença entre as médias Pós/Pré; * $p<0,05$. 
Apesar da utilização de frases-veículo sem conteúdo semântico, ao observar os espectros das frases (Figuras 3, 4, 5, 6 e 7), nota-se que os sujeitos conferiram rica modulação à leitura.

Não houve correlação estatisticamente significante entre a avaliação perceptivo-auditiva e a análise acústica.

Optou-se por considerar os próprios sujeitos como controle de si mesmos. Desta forma, foram automaticamente consideradas variáveis como: sexo, idade, tempo de profissão, quantidade de horas/aula semanais, quantidade média de alunos nas salas que ministram aula por período, nível de ensino por período, condições acústico-ergonômicas das salas de aula, atividade extraclasse, atividade de lazer que envolva uso vocal, entre outras.

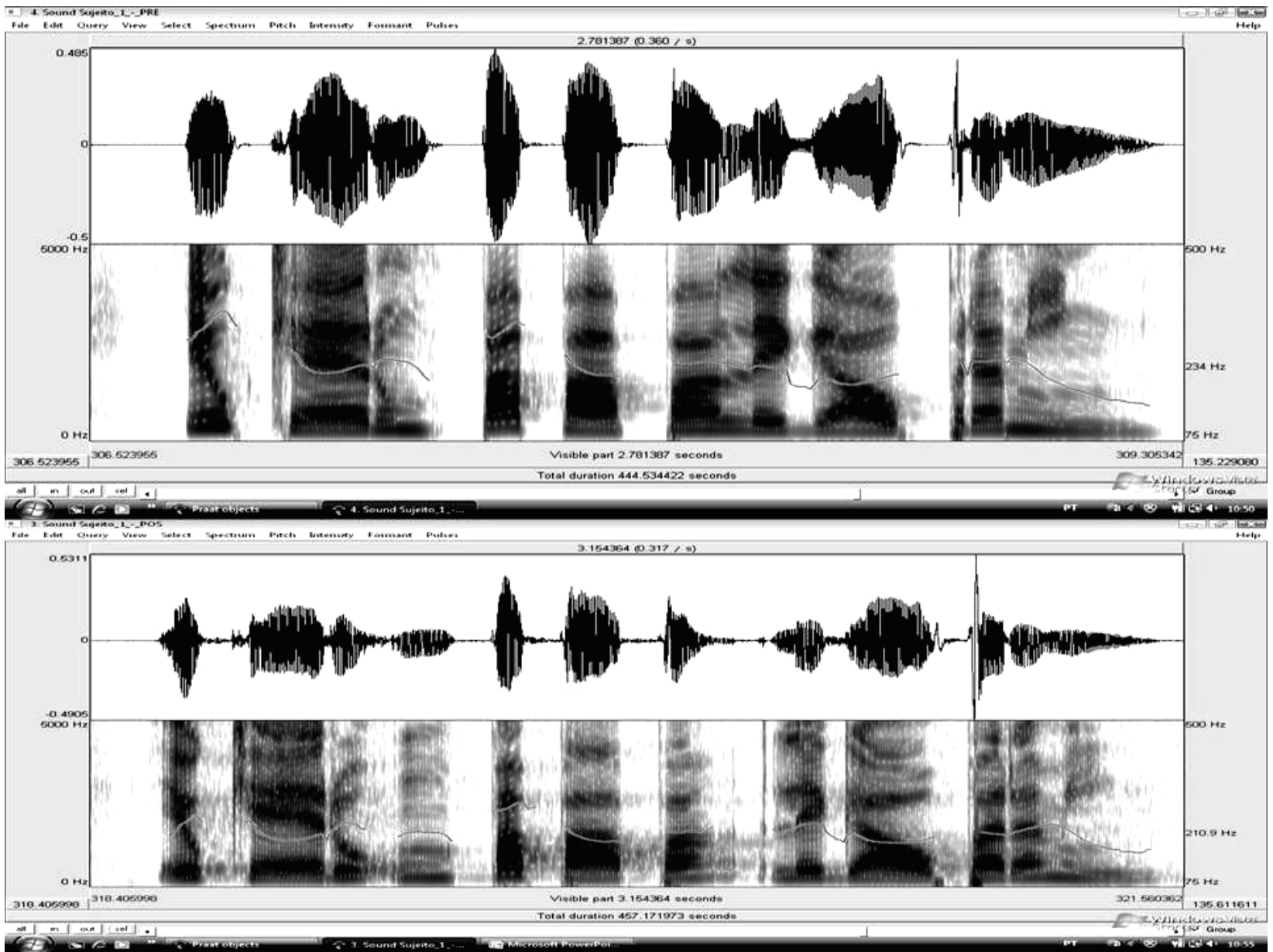

Fonte: software Praat versão 5028.

Figura 3 - Espectro de banca estreita da emissão do sujeito 1, com destaque para a variação de pitch na primeira repetição da frase "Eu quero um /pa'papa/ legal para mim", pré e pós-aprimoramento vocal, respectivamente.

\section{DISCUSSÃO}

O programa desenvolvido pelo presente estudo teve como principal objetivo o aprimoramento vocal e não o tratamento de alterações vocais. No entanto, houve adesão de professores com queixas vocais e/ou alterações laríngeas, o que tornou a intervenção preventivo-terapêutica. Após a participação no programa, os três sujeitos com alterações laríngeas foram encaminhados para terapia fonoaudiológica.

A baixa adesão de professores a estudos longitudinais é também observada por outros autores ${ }^{3,6,14}$. Apesar do número elevado de professores e da alta incidência de alterações vocais nessa classe profissional, a adesão a programas preventivos de 


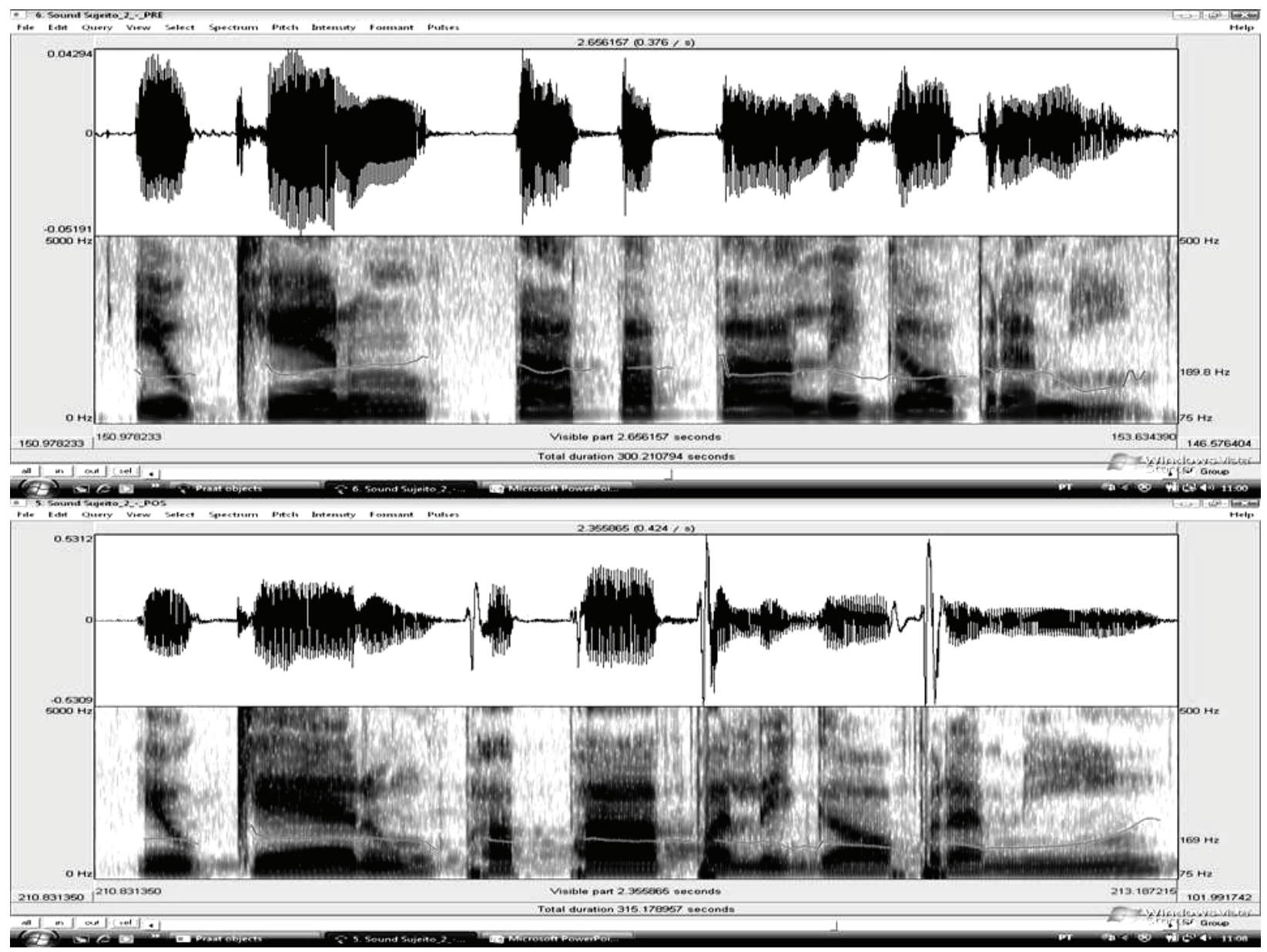

Fonte: software Praat versão 5028.

Figura 4 - Espectro de banca estreita da emissão do sujeito 2, com destaque para a variação de pitch na primeira repetição da frase "Eu quero um /pa'papa/ legal para mim", pré e pós-aprimoramento vocal, respectivamente.

longo prazo acaba sofrendo influência de diversos fatores, como por exemplo, não ter tempo hábil para participar de intervenções por trabalhar em mais de uma escola.

Além disso, a literatura revela que infelizmente a maioria dos professores apenas busca ajuda, quando a alteração vocal está impactando negativamente na sua atuação ${ }^{6}$. Professores que participaram de um grupo terapêutico realizado na Finlândia ${ }^{14}$ relataram como principal motivo para a não participação na intervenção, a ausência de alteração vocal. Pesquisadores acreditam que cursos de aprimoramento vocal deveriam ser oferecidos durante a formação profissional, para motivar e proporcionar uma maior participação $0^{3,14}$. Vale lembrar que mesmo sem queixa, alterações vocais e/ou laríngeas podem estar presentes ${ }^{22}$, e por isso a atenção à saúde vocal é de extrema importância para esta classe profissional.
Dada a importância da articulação precisa e das variações de freqüência e intensidade para a atuação vocal do professor, elegeram-se os parâmetros articulação, freqüência fundamental $e$ modulação de freqüência para as análises pré e pós-programa.

De acordo com a literatura ${ }^{23}$, os sujeitos apresentaram medidas de $\mathrm{f}_{0}$, tanto pré quanto pósintervenção, dentro do esperado para a população feminina brasileira, e pitch com média acima de nove na escala analógica visual. Desse modo, não era esperada mudança nestes parâmetros. A medida de $\mathrm{f}_{0}$, número de vibrações por segundo produzidas pelas pregas vocais, para mulheres é de $205 \mathrm{~Hz}$, podendo variar entre 150 e $250 \mathrm{~Hz}^{23}$. $\mathrm{Na}$ literatura há relato de variações de $\mathrm{f}_{0}$, aumento $e$ diminuição, em sujeitos pós-tratamento de disfonia funcional, mas todas se mantiveram dentro da faixa de normalidade ${ }^{24}$. 


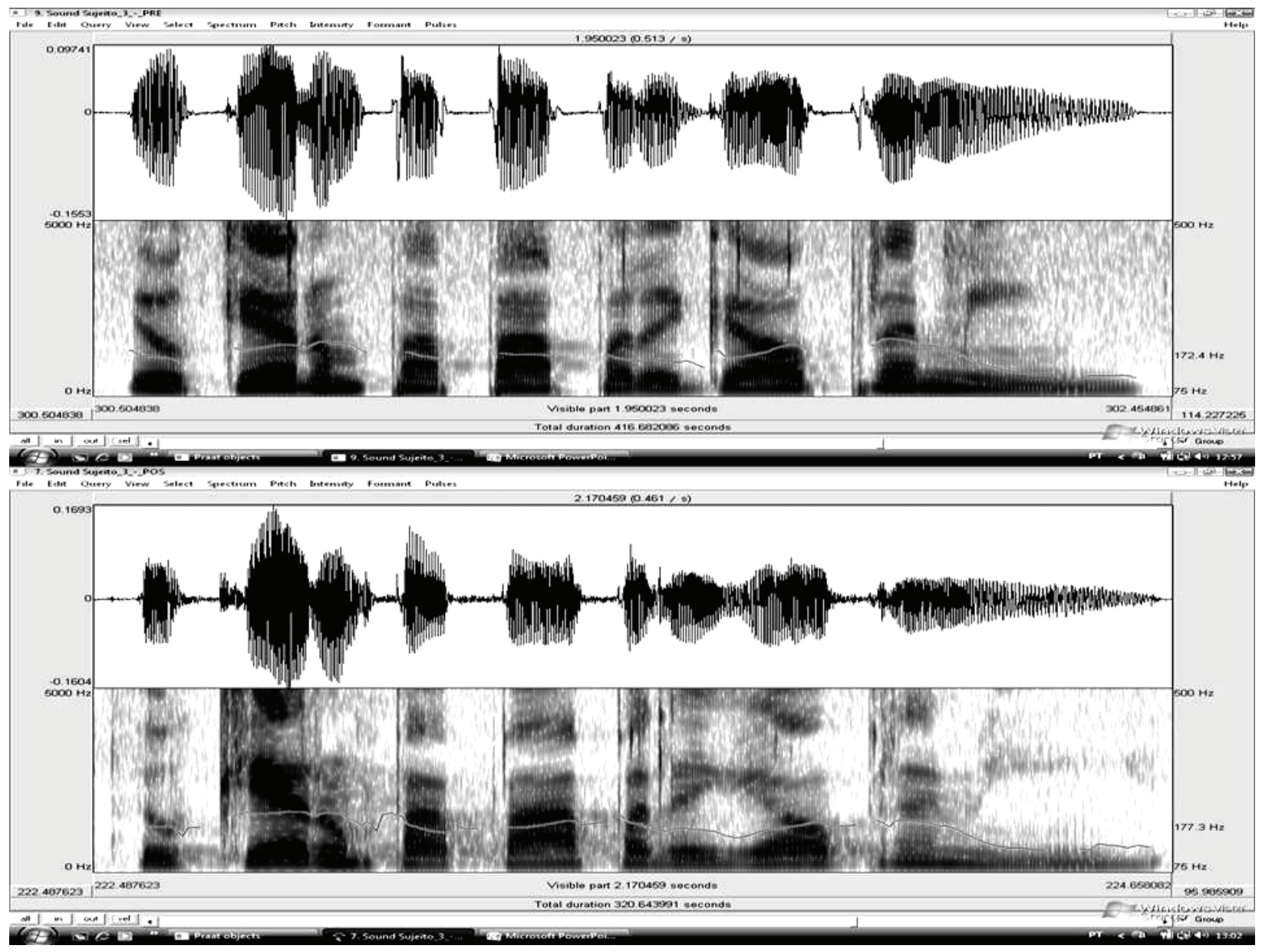

Fonte: software Praat versão 5028.

Figura 5 - Espectro de banca estreita da emissão do sujeito 3, com destaque para a variação de pitch na primeira repetição da frase "Eu quero um /pa'papa/ legal para mim", pré e pós-aprimoramento vocal, respectivamente.

Apesar da utilização de frases-veículo sem conteúdo semântico, ao observar os espectros das frases, nota-se que os sujeitos conferiram rica modulação à leitura. $O$ valor médio da modulação na escala analógica visual foi maior que oito, com aumento pós-intervenção, além da ampliação significante da extensão de freqüência, o que indica maior uso deste recurso expressivo pós-aprimoramento. Segundo a literatura, uma voz com modulação rica auxilia na transmissão da mensagem, diminui a probabilidade de fadiga vocal ${ }^{25}$ e permite maior facilidade no aprendizado do conteúdo escolar. De acordo com uma pesquisa realizada na Inglaterra, a voz do professor interfere no desempenho dos alunos, principalmente nas habilidades de compreensão, análise e síntese auditiva ${ }^{12}$.

Os valores de $F_{1}$ e $F_{2}$ encontrados neste estudo estão dentro do esperado. A literatura registra na vogal /a/ para mulheres valores de $F_{1}$ e $F_{2}$ próximos de $956 \mathrm{~Hz}$ e $1634 \mathrm{~Hz}$, respectivamente. Para a vogal /i/, valores próximos de $425 \mathrm{~Hz}$ e $2984 \mathrm{~Hz}$, e para a vogal /u/, valores próximos de $462 \mathrm{~Hz}$ e $1290 \mathrm{~Hz}^{22}$.

Com relação à articulação, os resultados mais significantes deste estudo se referem ao aumento de $F_{1}$ para duas $(/ \mathrm{i} /, / \mathrm{u} /)$ das três vogais analisadas (/a/, /i/, /u/). Tendo em vista que a articulação da fala consiste no processo de ajustes motores dos órgãos fonoarticulatórios, e que $F_{1}$ corresponde à movimentação da língua no plano vertical, sendo influenciado pela elevação e pelo abaixamento da mandíbula ${ }^{21}$, o aumento de $F_{1}$ indica maior abertura de boca, sugerindo melhora no ajuste articulatório pós-intervenção. A literatura mostra que a principal ferramenta de articulação para aumentar $F_{1}$ é o abaixamento da mandíbula, quanto maior este abaixamento, maior $F_{1}{ }^{26}$. Acredita-se que 0 aumento da movimentação da língua para baixo 


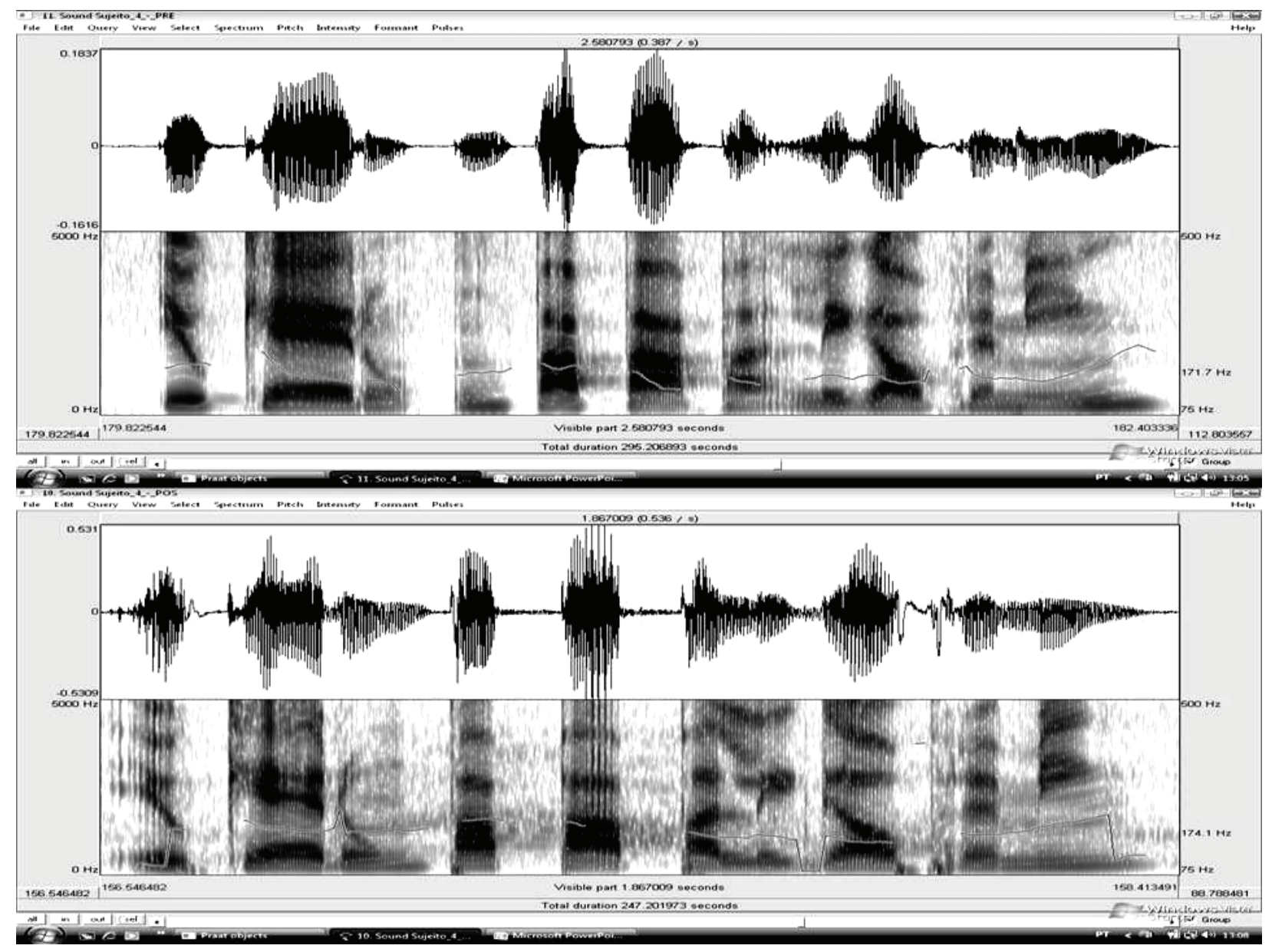

Fonte: software Praat versão 5028.

Figura 6 - Espectro de banca estreita da emissão do sujeito 4, com destaque para a variação de pitch na primeira repetição da frase "Eu quero um /pa'papa/ legal para mim", pré e pós-aprimoramento vocal, respectivamente.

mostrou-se mais evidente nas vogais /i/ e /u/ pois, no plano vertical a língua se posiciona baixa para a vogal /a/ e alta para as vogais /i/ e /u/.

Um aumento na abertura de boca pós-grupo de orientação e terapia vocal com professores ${ }^{2}$ e outros profissionais ${ }^{27}$ também foi observado na literatura. É importante destacar que uma fala com intensidade vocal, freqüência e articulação adequada, favorece a compreensão do aluno e diminui o esforço larínge ${ }^{10}$. Estudo recente, realizado no Brasil com professores, mostrou relação significante entre rouquidão e abertura de boca limitada ${ }^{28}$.

Atribui-se importância aos resultados encontrados, principalmente, ao considerar que os itens modulação e articulação, foram focalizados em apenas um e dois encontros da intervenção, respectivamente (ANEXO I). Mesmo com um pequeno número de casos analisados (5), foram observados resultados positivos e de impacto no uso profissional da voz.

Correspondência entre avaliação perceptivoauditiva e análise acústica foi encontrada por pesquisadores para alguns parâmetros de perturbação da onda sonora ${ }^{29}$. No entanto para os parâmetros avaliados neste estudo não pôde ser observada correspondência significante.

Destaca-se como prováveis limitações do estudo o número pequeno de sujeitos participantes, o não treinamento dos fonoaudiólogos avaliadores para a realização da análise acústico-perceptiva e a baixa sensibilidade dos parâmetros fonoarticulatórios selecionados. 


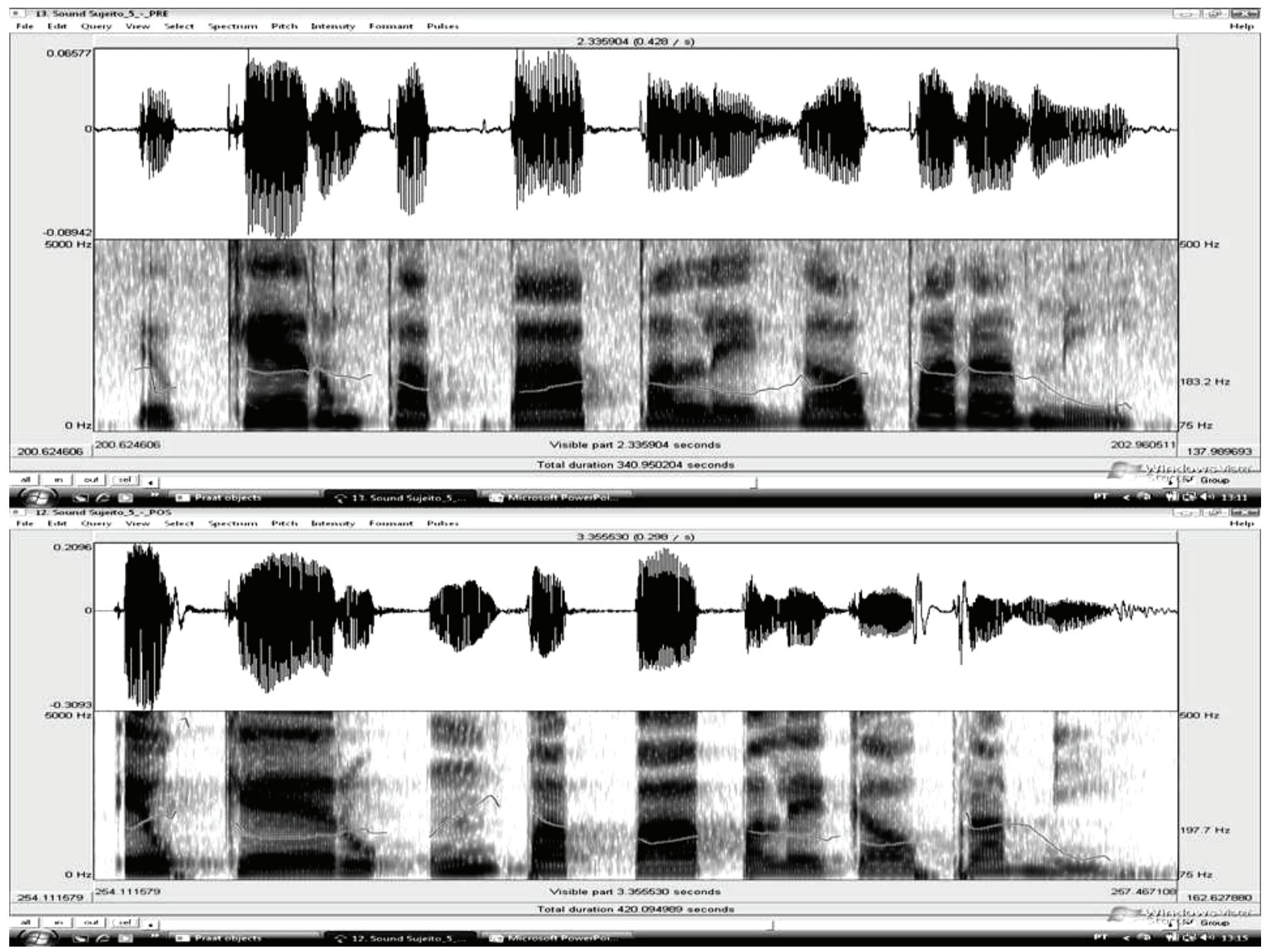

Fonte: software Praat versão 5028.

Figura 7 - Espectro de banca estreita da emissão do sujeito 5, com destaque para a variação de pitch na primeira repetição da frase "Eu quero um /pa'papa/ legal para mim", pré e pós-aprimoramento vocal, respectivamente

\section{CONCLUSÕES}

Os resultados do presente estudo, mesmo com um pequeno número de sujeitos, sugerem ampliação significante da extensão de freqüência e aumento estatisticamente significante de $F_{1}$ nas vogais /i/ e /u/ pós-intervenção.

Não foram observadas modificações no pitch, modulação e articulação avaliados por meio da análise perceptivo-auditiva, bem como da freqüência fundamental pós-intervenção.
Acredita-se que, o programa desenvolvido pode melhorar o uso profissional da voz. Logo, é valido aprofundarem-se os estudos, procurando aplicar este programa com um número maior de sujeitos, culminando com a adoção de programas preventivos desta natureza dentro da política de desenvolvimento profissional de professores. Só assim, os professores terão o tempo, a motivação e a dedicação necessários para aderirem a um programa preventivo desta natureza. 


\begin{abstract}
Purpose: to analyze and correlate teachers' vocal and articulation parameters in a pre and post vocal improvement program. Method: a vocal improvement program was offered to a fundamental education state school, located in Campinas, São Paulo, Brazil. Thirteen teachers agreed to take part in the program, however, due to the exclusion criterion, only five "subjects" were selected for the analysis. Teachers were previously submitted to a laryngologist evaluation. Twelve weekly meetings of one and a half hour each were held in the school. In the first and the last meetings, the teachers made a three sentence-vehicle recording, which were submitted to a perceptual evaluation (pitch, modulation and articulation) and an acoustic analysis (fundamental frequency, the frequency length and two first formants' assessment). Data were submitted to statistic analysis. Results: even with a small number of subjects, the results of this study, suggest significant expansion in the frequency extension and a statistically significant increase of $\mathrm{F} 1$ in the post-intervention vowels / $\mathrm{i} /$ and / $\mathrm{u} /$. Conclusions: there were no changes in pitch, modulation and articulation measured through perceptual analysis, and fundamental post-intervention frequency.
\end{abstract}

KEYWORDS: Voice; Faculty; Occupational Health; Voice Training; Disease Prevention

\section{REFERÊNCIAS}

1. Araújo TM, Reis EJFB, Carvalho FM, Porto LA, Reis IC, Andrade JM. Fatores associados a alterações vocais em professoras. Cad Saúde Pública 2008; 24(6):1229-38.

2. Bovo R, Galceran, Petruccelli J, Hatzopoulos S. Vocal problems among teachers: evaluation of a preventive voice program. J Voice 2007; 21(6):705-22.

3. Duffy OM, Hazlett DH. The impacto of preventive voice care programs for training teachers: a longitudinal study. J Voice 2004; 18(1):63-70.

4. Gillivan-Murphy P, Drinnan MJ, Dwyer TPO, Rdha $\mathrm{H}$, Carding P. The effectiveness of a voice treatment approach for teachers with self-reported voice problems. J Voice 2006; 20(3):423-31.

5. Grillo MHMM, Lima EF, Ferreira LP. A questão ensino aprendizagem num trabalho profilático de aperfeiçoamento vocal com professores. Pró-fono Rev 2000; 12(2):73-80.

6. Grillo MHMM. The impacto of a vocal improvement course in a speech language and hearing science prevention context. Pró-fono Rev 2004; 16(2);159-68.

7. Kooijman PGC, Jong FICRS, Thomas G, Huinck W, Donders R, Graamans K, Schutte HK. Risk factors for voice problems in teachers. Folia Phoniatr Logop 2006;58:159-74.

8. Kooijman PGC, Thomas G, Graamans K, Jong FICRS. Psychosocial impact of the teacher's voice throughout the career. J Voice 2007; 21(3):316-24.

9. Mattiske JA, Oates JM, Greenwood KM. Vocal problems among teacher: a rewiew of prevalence, causes, prevention and treatment. J Voice 1998; 12(4): 489-99.

10. Niebudek-Bogusz E, Sznurowska-Prygocka B, Fiszer M, Kotylo P, Sinkiewicz, Modrzewska M, et al. The effectiveness of voice therapy for teachers with dysphonia. Folia Phoniatr Logop 2008;60:134-41.

11. Penteado RZ, Bicudo IMT. Qualidade de vida e saúde vocal de professores. Rev Saúde Pública 2007;41(2):236-43.

12. Rogerson J, Dodd B. Is there an effect of dysphonic teachers' voices on children's processing of spoken language? J Voice 2005;19(1):47-60.

13. Simberg S, Sala E, Vehmas K, Laine A. Changes in the prevalence of vocal symptoms among teachers during a twelve-year period. J Voice 2005;19(1):95-102.

14. Simberg S, Sala E, Tuomainen J, Sellman J, Rönnemaa AM. The effectiveness of group therapy for students with mild voice disorders: a controlled clinical trial. J Voice 2006;20(1):97-109.

15. Tavares ELM, Martins RHG. Vocal evaluation in teachers with or without symptoms. J Voice 2007;21(4):407-14.

16. Yiu EML. Impact and prevention of voice problems in the teaching profession: embracing the cosumers' view. J Voice 2002; 16(2):215-28.

17. Roy N, Merrill RM, Thibealt S, Parsa RA, Gray $\mathrm{SD}$, Smith EM. Prevalence of voice disorders in teachers and the general population. J Speech Lang Hear Res 2004; 47:281-93.

18. Simões M, Latorre MRDO. Prevalência de alteração vocal em educadoras e sua relação com a auto-percepção. Rev Saúde Pública 2006;40(6):1013-8. 
19. Smith E, Gray SD, Dove H, Kirchner L, Heras H. Frequency and effects of teachers' voice problems. J Voice 1997;11(1):81-7.

20. Behlau M, Madazio G, Feijó D, Azevedo R, Gielow I, Rehder MI. Aperfeiçoamento vocal e tratamento das disfonias. In: Behlau M (organizadora). Voz - 0 livro do especialista. Rio de Janeio: Revinter; 2005. p.409-564.V. 2.

21. Kent R, Read C. The acoustic analysis of speech. San Diego: Singular;1992.

22. Corazza VR, Silva VFC, Queija DS, Dedivitis RA, Barros APB. Correlação entre os achados estroboscópicos, perceptivo-auditivos e acústicos em adultos sem queixa vocal. Rev Bras Otorrinolaringol 2004; 70(1):30-4.

23. Russo I, Behlau M. Percepção de fala: análise acústica do português brasileiro. São Paulo: Lovise; 1993.

24. Roy $\mathrm{N}$, Hendarto $\mathrm{H}$. Revisiting the pitch controversy: changes in speaking fundamental frequency (SFF) after managemenet of functional disfonia. J Voice 2005;19:582-91.
25. Behlau M, Dragone MLS, Nagano L. Mecanismo de produção de voz e fala. In: A voz que ensina. Rio de Janeiro: Revinter; 2004. p.27-39.

26. Lindblom B, Sundberg J. Acoustical consequences of lip, tongue, jaw, and larynx movement. J Acoust Soc Am 1971; 50:1166-79.

27. Borrego MC, Gasparini G, Behlau M. The effects of a specific speech and language training program on students of a radio announcing course. J Voice 2007; 21(4):426-32.

28. Ferreira LP, Latorre MRDO, Giannini SPP, Ghirardi ACAM, Karmann DF, Silva EE, et al. Influence of Abusive Vocal Habits, hidration, mastication and slepp in the occurrence of vocal symptoms in teachers. J Voice 2010; 24(1):86-92.

29. Bhuta T, Patrick L, Garnett JD. Perceptual evaluation of voice quality and its correlation with acoustic measurements. J Voice 2004; 18(3):299-304.
http://dx.doi.org/10.1590/S1516-18462011005000135

RECEBIDO EM: 29/11/2010

ACEITO EM: 29/05/2011

Endereço para correspondência:

Karen Fontes Luchesi

Av. Tessália Vieira de Camargo, no 126

Cidade Universitária, Campinas - SP - Brasil

CEP: 13083-887

E-mail: karenluchesi@yahoo.com.br 